\title{
Stochastic comparison of point random fields
}

\author{
Hans-Otto Georgii and Torsten Küneth \\ Mathematisches Institut der Universität München \\ Theresienstr. 39, D-80333 München \\ E-mail: georgii@rz.mathematik.uni-muenchen.de
}

\begin{abstract}
We give an alternative proof of a point-process version of the FKG-HolleyPreston inequality which provides a sufficient condition for stochastic domination of probability measures, and for positive correlations of increasing functions.

Stochastic Domination, FKG inequality, Correlation inequalities, Gibbs distribution, Poisson point process.

AMS 1991 Subject Classification: Primary 60 G 55

SECONDARY $60 \mathrm{~K} 35 ; 82$ B 21
\end{abstract}

\section{Introduction and result}

The FKG-Holley-Preston inequality $[4,7,14]$ plays a major rôle in the analysis of probability measures on products of linearly ordered spaces which occur, for example, in the lattice models of Statistical Mechanics. It provides a condition of attraction between the coordinates under which any two increasing functions are positively correlated or, more generally, two probability measures are comparable in the stochastic order. An analog for point random fields was derived by Preston [15]. Similar to Holley's argument in [7], Preston gave a dynamical proof by constructing a suitable coupling of two ergodic spatial birth-and-death processes which leave the two considered point random fields invariant. The discrete result, however, also admits non-dynamical proofs which proceed by induction on the number of coordinates. (The simplest is that of Batty and Bollmann [2].) So one may ask whether such a "direct" proof can also be found in the continuous case of point random fields. It is the purpose of this note to provide such a non-dynamical proof. This proof is technically simpler and allows us to dispense with a technical condition needed by Preston to ensure the existence and ergodicity of the birth-and-death processes. As Janson [8] in his proof of an analog of Harris' inequality [6] for Poisson point processes (see Example 2.1 below), we will use a discretization argument which reduces the continuous case to the classical discrete case.

Let $E$ be a separable metric space with Borel $\sigma$-algebra $\mathcal{E}$. $E$ serves as state space for points, or particles, living on $E$. A configuration of finitely many particles in $E$ is 
conveniently described by an element of $\mathcal{M}=\mathcal{M}_{\mathrm{pf}}(E, \mathcal{E})$, the set of all finite integervalued measures on $(E, \mathcal{E})$ or, equivalently, of all finite sums of Dirac measures on $(E, \mathcal{E})$. Given a configuration $\mu \in \mathcal{M}$, we write $x \in \mu$ if $\mu(\{x\})>0$, i.e., if at least one particle of $\mu$ has position $x$. $\mathcal{M}$ is equipped with the $\sigma$-algebra $\mathcal{F}$ generated by the counting variables $N_{A}: \mu \rightarrow \mu(A)$ on $\mathcal{M}, A \in \mathcal{E}$. A probability measure $P$ on $(\mathcal{M}, \mathcal{F})$ is called a point random field (prf).

We will be concerned with the stochastic order between prf's which is induced by the usual order on $\mathcal{M}$. Specifically, for $\mu, \nu \in \mathcal{M}$ we write $\mu \leq \nu$ if $\mu(A) \leq \nu(A)$ for all $A \in \mathcal{E}$. (In the particle language, this means that $\nu$ has more particles than $\mu$.) A function $h: \mathcal{M} \rightarrow \mathrm{R}$ is called increasing if $h(\mu) \leq h(\nu)$ whenever $\mu \leq \nu$. Since the empty configuration $0 \in \mathcal{M}$ is minimal in $\mathcal{M}$, each increasing $h$ is bounded from below by $h(0)$. A set $B \subset \mathcal{M}$ is called increasing if its indicator function $1_{B}$ is increasing.

For any two prf's $P$ and $Q$, we say $Q$ dominates $P$ in the stochastic order, and write $P \preceq Q$, if $\int h d P \leq \int h d Q$ for all increasing (without loss bounded) measurable functions $h: \mathcal{M} \rightarrow$ R. By a celebrated theorem of Strassen (cf. Section IV.1.2 of [11]), for complete $E$ this is equivalent to the existence of a coupling of $P$ and $Q$ which is supported on the set $\{(\mu, \nu): \mu \leq \nu\}$.

We investigate this stochastic order relation for a class of prf's which are absolutely continuous with respect to a Poisson prf. Let $\lambda$ be a fixed finite reference measure on $(E, \mathcal{E})$ and $\mathbf{P}=\mathbf{P}_{\lambda}$ the Poisson prf with intensity measure $\lambda$. That is, $\mathbf{P}$ is the image of the probability measure $e^{-\lambda(E)} \sum_{n \geq 0} \frac{1}{n !} \lambda^{n}$ on the disjoint union $\bigcup_{n \geq 0} E^{n}$ under the mapping $\left(x_{1}, \ldots, x_{n}\right) \rightarrow \delta_{x_{1}}+\ldots+\delta_{x_{n}} \in \mathcal{M},\left(x_{1}, \ldots, x_{n}\right) \in E^{n}, n \geq 0$. (The unique element of $E^{0}$ with mass $e^{-\lambda(E)}$ is mapped to the empty configuration $0 \in \mathcal{M}$.)

Definition. (a) A prf $P$ belongs to the class $\mathcal{P}_{\lambda}$ if $P \ll \mathbf{P}$ and its density admits a version $f_{P}$ with increasing zero-set $\left\{f_{P}=0\right\}$.

(b) For each $P \in \mathcal{P}_{\lambda}$, any measurable function $\gamma_{P}: E \times \mathcal{M} \rightarrow[0, \infty$ [ satisfying

$$
f_{P}\left(\mu+\delta_{x}\right)=\gamma_{P}(x, \mu) f_{P}(\mu) \text { for all } x \in E, \mu \in \mathcal{M}
$$

is called (a version of) the Papangelou (conditional) intensity of $P$.

Obviously, the defining condition of $\mathcal{P}_{\lambda}$ that $\left\{f_{P}=0\right\}$ is increasing is necessary and sufficient for the existence of a Papangelou intensity $\gamma_{P}$. One version of $\gamma_{P}$ can be obtained by setting $\gamma_{P}(x, \mu)=f_{P}\left(\mu+\delta_{x}\right) / f_{P}(\mu)$ if $f_{P}(\mu)>0$ and $\gamma_{P}(x, \mu)=0$ otherwise. A different characterization of $\mathcal{P}_{\lambda}$ explaining the significance of the Papangelou intensity will be given in Proposition 3.1. In particular, we will see there that each $P \in \mathcal{P}_{\lambda}$ is uniquely determined by $\gamma_{P}$. Proposition 3.1 will also imply that the Papangelou intensity of a prf $P$ can be chosen independently of $\mu$ (i.e., $\gamma_{P}(x, \mu)=\rho(x)$ for all $x, \mu$ and a measurable function $\rho \geq 0$ ) if and only if $\rho$ is $\lambda$-integrable and $P=\mathbf{P}_{\rho \lambda}$, the Poisson prf with intensity measure $\rho(x) \lambda(d x)$. The following continuous analog of the FKG-Holley-Preston inequality is the main result of this paper. 
Theorem 1.1 Suppose $P, Q \in \mathcal{P}_{\lambda}$ admit Papangelou intensities $p=\gamma_{P}$ and $q=\gamma_{Q}$ satisfying

$$
p(x, \mu) \leq q(x, \nu) \text { whenever } \mu \leq \nu \text { and } x \notin \nu-\mu,
$$

$\mu, \nu \in \mathcal{M}, x \in E$. Then $P \preceq Q$.

Intuitively, condition (2) means that it is more likely to add a particle to a typical configuration $\nu$ of $Q$ than to a smaller configuration $\mu$ which is typical for $P$. In other words, the $\operatorname{prf} Q$ exhibits a stronger attraction between the particles than $P$. This is seen most clearly in the case $P=Q$ in which condition (2) can be circumscribed as follows: the more particles are present, the more likely is the appearence of a further particle. This special case gives us the following point-process version of the FKGinequality.

Corollary 1.2 Let $P \in \mathcal{P}_{\lambda}$, and suppose $P$ is attractive in the sense that $\gamma_{P}(x, \cdot)$ is increasing on $\left\{N_{\{x\}}=k\right\}$ for all $x \in E$ and $k \geq 0$. Then $P$ has positive correlations, in that

$$
\int g h d P \geq \int g d P \quad \int h d P
$$

for any two increasing bounded measurable functions $g$ and $h$ on $\mathcal{M}$.

Proof. We can assume without loss of generality that $g>0$. (Otherwise we replace $g$ by $g+c$ for a suitable constant $c$.) The probability measure $Q(d \nu)=$ $g(\nu) P(d \nu) / \int g d P$ has then the Papangelou intensity $q(x, \nu)=p(x, \nu) g\left(\nu+\delta_{x}\right) / g(\nu)$, and (2) holds for $p$ and $q$. Hence $P \preceq Q$, which gives the result.

In the next section we will discuss a few examples. In Section 3 we introduce the 'smallest' (resp. 'largest') prf whose Papangelou intensity lies above (resp. below) a given function, and investigate their properties. These are our main tool for the proof of Theorem 1.1 in Section 4.

We conclude this introduction with several remarks.

Remark 1.3 Let $P, Q \in \mathcal{P}_{\lambda}$ with $\mathbf{P}$-densities $f=f_{P}, g=f_{Q}$. Condition (2) then implies that

$$
f(\mu) g(\nu) \leq f(\mu \wedge \nu) g(\mu \vee \nu) \text { for all } \mu, \nu \in \mathcal{M}
$$

which corresponds directly to the condition of the classical FKG-Holley-Preston inequality. Indeed, (3) can be rewritten in the form

$$
f(\mu+\alpha) g(\nu) \leq f(\mu) g(\nu+\alpha) \text { for all } \alpha, \mu, \nu \in \mathcal{M} \text { with } \mu \leq \nu, \alpha \perp \nu-\mu .
$$

But this property follows readily from (1) and (2) by induction on $\alpha(E)$. In fact, we see that (2) and (3) are equivalent when $g>0$ (provided we choose a version of $p$ with $p(x, \cdot)=0$ on $\{f=0\}$.

Remark 1.4 Suppose $E$ is finite. Then $\mathcal{M}$ can be identified with the product space $\mathrm{N}^{E}$, where $\mathrm{N}$ is the set of all nonnegative integers. Accordingly, $\mathbf{P}$ can be identified 
with the product of the Poisson distributions with parameters $\lambda(\{x\}), x \in E$. Together with the preceding remark this shows that, for finite $E$, Theorem 1.1 is a special case of the classical Holley-Preston inequality. We shall prove Theorem 1.1 by reducing it to the case of finite $E$ by means of a suitable discretization.

REMARK 1.5 Although condition (2) is the direct analog of the classical HolleyPreston condition, its usefulness is much more limited. This is because, in the continuous case, the particle attraction expressed by (2) interferes with the necessary requirement of stability which prevents the occurrence of infinitely many particles in $E$. A first indication of this conflict is the following observation which follows readily from (9) below. If condition (2) holds and $P$ is nontrivial on a set $A \in \mathcal{E}$, in that $P\left(N_{A}>0\right)>0$, then $Q\left(N_{A}=n\right)>0$ for all $n$. In other words, unless $P$ is degenerate, the dominating measure $Q$ is not allowed to exhibit any kind of hard-core exclusion between the particles. We shall return to this point in Example 2.2 below.

REMARK 1.6 In addition to (2) and the monotonicity of the zero-sets of the $\mathbf{P}$ densities of $P$ and $Q$, Preston [15] needs a decay condition on the numbers $\beta_{n}=$ $\sup _{\mu \in \mathcal{M}: \mu(E)=n} \int \gamma_{Q}(\cdot, \mu) d \lambda$ in order to ensure the existence and ergodicity of the spatial birth-and-death processes with invariant measures $P$ resp. $Q$. On p. 389, he tries to weaken this condition using an approximation argument. However, one can show by example that the approximating functions may fail to satisfy the required decay condition. So one has to resort to refined approximations of the form we use in Section 4.

\section{Examples}

In spite of the conflict between attractiveness and stability which we discussed in Remark 1.5, there exists a number of possible applications of Theorem 1.1 and Corollary 1.2. These are the subject of this section.

Example 2.1 Poisson prf's. The hypothesis of attractivity in Corollary 1.2 is certainly satisfied when $\gamma_{P}=1$, i.e., if $P=\mathbf{P}$. This shows that each Poisson prf has positive correlations. This result, which was obtained first by Janson [8], is the pointprocess analog of an inequality of Harris [6] which states that every Bernoulli measure has positive correlations.

A further simple application of Theorem 1.1 is the following "Poisson sandwich inequality". If $P \in \mathcal{P}_{\lambda}$ is such that

$$
\rho_{1}(x) \leq \gamma_{P}(x, \mu) \leq \rho_{2}(x)
$$

for all $x \in E, \mu \in \mathcal{M}$ and suitable $\lambda$-integrable functions $\rho_{i} \geq 0$ on $\mathcal{M}$ then

$$
\mathbf{P}_{\rho_{1} \lambda} \preceq P \preceq \mathbf{P}_{\rho_{2} \lambda} .
$$


We note that this conclusion can also be deduced from the positive correlations of the Poisson prf's $\mathbf{P}_{\rho_{i} \lambda}$. Indeed, if we assume for simplicity that $\rho_{i}>0$ then condition (4) simply means that the functions

$$
g_{i}(\mu)=f_{P}(\mu) \exp \left[-\int \log \rho_{i} d \mu\right]
$$

are increasing (for $i=1$ ) resp. decreasing (for $i=2$ ), and $g_{i}$ is the $\mathbf{P}_{\rho_{i} \lambda}$-density of $P$ up to a constant factor. (A version of (5) appears also in [3].)

A straightforward extension of Janson's result above concerns the positive correlations of mixed Poisson prf's. Suppose $I$ is an interval in $\mathrm{R}$ and $\rho: I \times E \rightarrow[0, \infty[$ a measurable function such that $\rho\left(z_{1}, \cdot\right) \leq \rho\left(z_{2}, \cdot\right)$ when $z_{1} \leq z_{1}$ and each $\rho(z, \cdot)$ is $\lambda$-integrable. Let $P_{z}=\mathbf{P}_{\rho(z, \cdot) \lambda}$ and $P=\int w(d z) P_{z}$ for some probability measure $w$ on $I$. Then $P$ has positive correlations. For, if $g, h$ are increasing bounded measurable functions on $\mathcal{M}$ then

$$
\begin{aligned}
\int g h d P & \geq \int w(d z)\left(\int g d P_{z}\right)\left(\int h d P_{z}\right) \\
& \geq \int g d P \int h d P .
\end{aligned}
$$

The first inequality comes from the positive correlations of the $P_{z}$ 's. To see the second inequality we note that, by Theorem 1.1, $P_{z_{1}} \preceq P_{z_{2}}$ when $z_{1} \leq z_{2}$. The functions $z \rightarrow \int g d P_{z}$ and $z \rightarrow \int h d P_{z}$ are therefore increasing, and it is well-known [14] that every probability measure $w$ on a set $I$ with a measurable linear order has positive correlations.

Next we turn to prf's with interaction between the particles. Recall that the Gibbs distribution for a measurable "Hamiltonian" $H: \mathcal{M} \rightarrow \mathrm{R} \cup\{\infty\}$ with

$$
0<Z \equiv \int e^{-H} d \mathbf{P}<\infty
$$

is defined as the prf $G_{H}$ with $\mathbf{P}$-density $Z^{-1} e^{-H}$. If $\{H=\infty\}$ is increasing, $G_{H}$ belongs to the class $\mathcal{P}_{\lambda}$, and its Papangelou intensity satisfies

$$
\gamma_{H}(x, \mu)=\exp \left[-H\left(\mu+\delta_{x}\right)+H(\mu)\right] \text { when } H(\mu)<\infty,
$$

$x \in E, \mu \in \mathcal{M}$.

EXAMPLe 2.2 Gibbs distributions with pair interaction. The standard type of Hamiltonian has the form

$$
H(\mu) \equiv H_{u, v}(\mu)=\frac{1}{2} \int \mu(d x) \int\left(\mu-\delta_{x}\right)(d y) u(x, y)+\int \mu(d x) v(x) .
$$

Here $u: E \times E \rightarrow \mathrm{R} \cup\{\infty\}$ is a symmetric measurable function, the pair potential, and $v: E \rightarrow \mathrm{R} \cup\{\infty\}$ a measurable chemical potential that might be induced by the interaction with a fixed boundary condition. (That is, $v=\int \alpha(d y) u(\cdot, y)$ for a fixed 
configuration $\alpha$.) A natural version of the associated Papangelou intensity (6) is given by

$$
\gamma_{u, v}(x, \mu)=\exp \left[-v(x)-\int \mu(d y) u(x, y)\right] 1_{\mathcal{M}_{u}}(\mu),
$$

where $\mathcal{M}_{u}=\left\{\mu \in \mathcal{M}: u(x, y)<\infty\right.$ whenever $\left.\delta_{x}+\delta_{y} \leq \mu\right\}$ is the set of admissible configurations. Let us investigate under which conditions Theorem 1.1 is applicable. So let $\tilde{u}, \tilde{v}$ be a second pair of potential functions and suppose $p=\gamma_{u, v}$ and $q=\gamma_{\tilde{u}, \tilde{v}}$ satisfy (2). We can assume without loss of generality that $\tilde{v}=0$ because otherwise $\lambda$ can be replaced by $e^{-\tilde{v}} \lambda$. We also assume for simplicity that $v<\infty$. (Otherwise we can confine ourselves to the set $\{v<\infty\}$ by means of Remark 3.2.) Setting $\mu=0$ and $\nu=n \delta_{y}$ in (2) with arbitrary $n \geq 1$ and $y \in E$ we then see that $\tilde{u} \leq 0$. On the other hand, it is impossible that $\int \tilde{u} d \lambda^{2}<0$ because then the normalizing constant $\tilde{Z}=\int e^{-H_{\tilde{u}}, 0} d \mathbf{P}$ would be infinite, cf. Proposition 3.2.4 of [16]. Hence $\tilde{u}=0$ up to $\lambda^{2}$-nullsets. This means that $G_{\tilde{u}, 0}=\mathbf{P}$. In other words, if two Gibbs distributions with pair interaction satisfy (2), the dominating measure must necessarily be a Poisson prf. Moreover, (2) then reduces to the requirement that $\gamma_{u, v} \leq 1$ or, equivalently, that $\int \mu(d y) u(\cdot, y) \geq-v$ for all $\mu \in \mathcal{M}_{u}$. There are only two cases of interest where the last requirement can be fulfilled, namely

(i) $u \geq 0$ (the case of pure repulsion), and

(ii) $u$ has a hard core, in that $u(x, y)=\infty$ when $d(x, y) \leq 1$ for a suitable metric $d$ on $E$, and $u \geq-\psi \circ d$ for a decreasing function $\psi:[0, \infty[\rightarrow[0, \infty$ [ which decays to zero sufficiently rapidly.

In both cases we can conclude that the Gibbs distributions are dominated by suitable Poisson prf's. But this is the only possible application of Theorem 1.1 to Gibbs distributions with pair interaction.

Since a stable pair interaction can never be attractive, one may ask whether the conflict between attractivity and stability can be solved by multi-particle interactions. Adding only a triple interaction is not sufficient because attractivity would also force the triple interaction to be nonpositive. In fact, it is necessary to include $n$-tuple interactions for arbitrary $n$. (Note that, by the Möbius inversion, every Hamiltonian can be expressed as a sum of $n$-tuple contributions.) The next example shows that a stable Hamiltonian with an appropriate balance between the $n$-tuple interactions may indeed be attractive.

EXAMPLE 2.3 Interactions of Widom-Rowlinson type. Imagine a system of particles of two types (A and B) with a repulsive pair interaction $u \geq 0$ between particles of different type and no interaction between particles of the same type. The marginal distribution of the A-particles is then Gibbsian with a Hamiltonian of the form

$$
H(\mu) \equiv H_{u, v}^{A}(\mu)=\int \lambda(d y)\left(1-\exp \left[-v(y)-\int \mu(d x) u(x, y)\right]\right),
$$


$\mu \in \mathcal{M}$; see [5], for example. (The function $v: E \rightarrow \mathrm{R} \cup\{\infty\}$ takes account of a possible interaction between the B-particles and a fixed boundary condition of A-particles.) In the special case when $v=0$ and $u(x, y)=\infty$ when $d(x, y) \leq 1$ and 0 otherwise, we obtain the Hamiltonian $H(\mu)=\lambda\left(\bigcup_{x \in \mu} B(x)\right)$ introduced by Widom and Rowlinson [17]; here $B(x)$ is the unit ball centered at $x$. (This case is also referred to as the area-interaction model [1].) The Papangelou intensity associated to (7) is equal to

$$
\gamma_{u, v}^{A}(x, \mu)=\exp \left\{-\int \lambda(d y)\left(1-e^{-u(x, y)}\right) \exp \left[-v(y)-\int \mu(d z) u(y, z)\right]\right\} .
$$

Since $u \geq 0$, this function satisfies the attractivity condition of Corollary 1.2. We thus conclude that the Gibbs distribution $G_{u, v}^{A}$ with Hamiltonian (7) has positive correlations. (A related result was found earlier in [10].) Moreover, an application of Theorem 1.1 shows that $G_{u, v}^{A} \preceq G_{\tilde{u}, \tilde{v}}^{A}$ whenever $u \leq \tilde{u}$ and

$$
\left(1-e^{-u(\cdot, y)}\right) e^{-v(y)} \geq\left(1-e^{-\tilde{u}(\cdot, y)}\right) e^{-\tilde{v}(y)}
$$

for all $y \in E$. (This follows also from the positive correlations of $G_{u, v}^{A}$ since $d G_{\tilde{u}, \tilde{v}}^{A} / d G_{u, v}^{A}$ is increasing.) In particular we see that, for any $n$, each $G_{u, v}^{A}$ can be squeezed between $G_{u_{n}, v-\epsilon_{n}}^{A}$ and $G_{u^{n}, v+\epsilon_{n}}^{A}$ with the bounded potential $u_{n}=u \wedge n$, the hard-core potential $u^{n}=u+\infty 1_{\{u>n\}}$, and $\epsilon_{n}=-\log \left(1-e^{-n}\right)$. A less stringent sandwich property is obtained from (5), namely $\mathbf{P}_{\rho \lambda} \preceq G_{u, v}^{A} \preceq \mathbf{P}_{\lambda}$ with

$$
\rho(x)=\exp \left\{-\int\left(1-e^{-u(x, \cdot)}\right) e^{-v} d \lambda\right\} .
$$

EXAMPLE 2.4 The continuous random cluster model. Let $E$ be a finite box in $\mathrm{R}^{d}$ and $\lambda=z \times($ Lebesgue measure on $E$ ) for a number $z>0$. Since $\lambda$ is diffuse, $\mathbf{P}$ is simple. That is, $\mathbf{P}$-almost all configurations $\mu$ have no multiple points and can thus be viewed as finite subsets of $E$. Given such a $\mu$, we draw an edge between any two distinct points $x, y \in \mu$ with $|x-y| \leq 1$. Let $k(\mu)$ be the number of components of the resulting graph. For any $s \geq 1$, the probability measure $P_{s}$ with $\mathbf{P}$-density proportional to $s^{k(\mu)}$ is called the continuous random cluster measures on $E$ (with free boundary condition).

The interest in $P_{s}$ comes from the following fact. Suppose $s$ is an integer, and we assign to each $x \in \mu$ a "type" $\sigma_{x} \in\{1, \ldots, s\}$ as follows. In a first step, all connected components of $\mu$ get independent equidistributed types, and then each particle $x \in \mu$ obtains the type of the component to which it belongs. If $\mu$ is distributed according to $P_{s}$, the resulting joint distribution of the pair $\left(\mu,\left(\sigma_{x}\right)_{x \in \mu}\right)$ is the Gibbs distribution for particles of $s$ different types with hard-core exclusion between unlike particles of distance $\leq 1$. (The case $s=2$ corresponds to the Widom-Rowlinson model considered in Example 2.3.)

Now, as was observed independently by [3] and both authors of [5], $P_{s} \in \mathcal{P}_{\lambda}$ with Papangelou intensity $s^{1-\kappa(x, \mu)}$, where $\kappa(x, \mu)$ is the number of connected components of $\mu$ which meet the ball with diameter 1 centered at $x$. For geometric reasons, $\kappa(x, \mu) \leq c$ 
for a constant $c<\infty$ depending on the dimension $d$. (5) therefore implies that $P_{s} \succeq \mathbf{P}_{b \lambda}$ with $b=s^{1-c}$. This stochastic domination implies the following. As $\mathbf{P}_{b \lambda}$ exhibits the phenomenon of percolation when $z$ is large enough [13], the same must also be true for $P_{s}$. But from this one can easily conclude that the associated multi-type particle system with intertype hard-core exclusion shows a phase transition. In [5], this line of reasoning is extended to prove a phase transition in multi-type particle systems with soft-core repulsion between unlike particles (as in the first sentence of Example 2.3). This amounts to considering a continuous random cluster model with random edges, and a key step is again the derivation of a lower bound on the Papangelou intensity to obtain a stochastic comparison with a Poisson prf.

\section{Papangelou intensities and the upper and lower prf's}

We start with a characterization of the class $\mathcal{P}_{\lambda}$ which clarifies the rôle of the Papangelou intensities. Recall that the reduced Campbell measure $\Gamma_{P}$ of a prf $P$ is defined on $(E \times \mathcal{M}, \mathcal{E} \otimes \mathcal{F})$ by

$$
\Gamma_{P}(C)=\int P(d \mu) \int \mu(d x) 1_{C}\left(x, \mu-\delta_{x}\right),
$$

$C \in \mathcal{E} \otimes \mathcal{F}$. The reduced Campbell measure of the Poisson prf $\mathbf{P}$ is given by $\boldsymbol{\Gamma} \equiv \Gamma_{\mathbf{P}}=$ $\lambda \otimes \mathbf{P}$, as is well-known [9] and easy to check.

Proposition 3.1 A prf $P$ belongs to the class $\mathcal{P}_{\lambda}$ if and only if $\Gamma_{P} \ll \lambda \otimes P$. In this case, any Papangelou intensity $\gamma_{P}$ of $P$ is a version of the Radon-Nikodym density $d \Gamma_{P} / d(\lambda \otimes P)$, and vice versa. One may choose a version of $\gamma_{P}$ which satisfies the cocycle property

$$
\gamma_{P}(x, \mu) \gamma_{P}\left(y, \mu+\delta_{x}\right)=\gamma_{P}(y, \mu) \gamma_{P}\left(x, \mu+\delta_{y}\right),
$$

$x, y \in E, \mu \in \mathcal{M}$, and $P$ is determined by $\gamma_{P}$ via the equation

$$
f_{P}\left(\delta_{x_{1}}+\ldots+\delta_{x_{n}}\right)=f_{P}(0) \gamma_{P}\left(x_{1}, 0\right) \gamma_{P}\left(x_{2}, \delta_{x_{1}}\right) \ldots \gamma_{P}\left(x_{n}, \delta_{x_{1}}+\ldots+\delta_{x_{n-1}}\right),
$$

$n \geq 1, x_{1}, \ldots, x_{n} \in E$.

In the point process literature, the condition $\Gamma_{P} \ll \lambda \otimes P$ is known as condition $\left(\Sigma_{\lambda}^{\prime}\right)$, see $[12,9]$.

Proof. We drop all indices referring to $P$.

"only if" Let $P \in \mathcal{P}_{\lambda}$. Using the identity $\Gamma_{\mathbf{P}}=\lambda \otimes \mathbf{P}$ we conclude from (1) that for each $C \in \mathcal{E} \otimes \mathcal{F}$

$$
\begin{aligned}
\Gamma(C) & =\int \mathbf{P}(d \mu) \int \mu(d x) f\left(\mu-\delta_{x}+\delta_{x}\right) 1_{C}\left(x, \mu-\delta_{x}\right) \\
& =\int \lambda(d x) \int \mathbf{P}(d \mu) f\left(\mu+\delta_{x}\right) 1_{C}(x, \mu) \\
& =\int \lambda(d x) \int P(d \mu) \gamma(x, \mu) 1_{C}(x, \mu) .
\end{aligned}
$$


Hence $\gamma$ is a version of $d \Gamma / d(\lambda \otimes P)$. On the other hand, (1) obviously implies (9) by induction on $n$, and (9) determines $f$ up to a constant which in turn is determined by the normalization of $f$. (8) follows by iteration from (1) if we set $\gamma(x, \cdot)=0$ on $\{f=0\}$.

"if" This is essentially due to [12]. We reproduce here the argument for later purposes. Let $\gamma$ be any version of $d \Gamma / d(\lambda \otimes P)$. By iteration we then find that the function $(x, y, \mu) \rightarrow \gamma(x, \mu) \gamma\left(y, \mu+\delta_{x}\right)$ is a density of the measure

$$
\int P(d \mu) \int \mu(d x) \int\left(\mu-\delta_{x}\right)(d y) 1\left\{\left(x, y, \mu-\delta_{x}-\delta_{y}\right) \in \cdot\right\}
$$

on $E \times E \times \mathcal{M}$ relative to $\lambda \otimes \lambda \otimes P$. Here we have written $1\{\ldots\}$ for the indicator function of the set $\{\ldots\}$. Since these two measures are symmetric in the two $E$-coordinates, it follows that $\gamma$ satisfies the cocycle property (8) for $\lambda \otimes \lambda \otimes P$-almost all $(x, y, \mu)$. Proceeding by induction on the number $n$ of $E$-coordinates and summing over $n$, we find that the function

$$
\begin{aligned}
& \varphi(0, \mu)=1 \\
& \varphi\left(\delta_{x_{1}}+\ldots+\delta_{x_{n}}, \mu\right)=\gamma\left(x_{1}, \mu\right) \gamma\left(x_{2}, \mu+\delta_{x_{1}}\right) \ldots \gamma\left(x_{n}, \mu+\delta_{x_{1}}+\ldots+\delta_{x_{n-1}}\right)
\end{aligned}
$$

on $\mathcal{M} \times \mathcal{M}$ is $\mathbf{P} \otimes P$-almost everywhere well-defined and - up to a factor of $e^{\lambda(E)}-$ a version of the Radon-Nikodym density of the reduced compound Campbell measure

$$
\begin{aligned}
\bar{\Gamma}_{P}= & \int P(d \mu) \sum_{n=0}^{\mu(E)} \frac{1}{n !} \int \mu\left(d x_{1}\right) \int\left(\mu-\delta_{x_{1}}\right)\left(d x_{2}\right) \ldots \\
& \int\left(\mu-\delta_{x_{1}}-\ldots-\delta_{x_{n-1}}\right)\left(d x_{n}\right) 1\left\{\left(\delta_{x_{1}}+\ldots+\delta_{x_{n}}, \mu-\delta_{x_{1}}-\ldots-\delta_{x_{n}}\right) \in \cdot\right\}
\end{aligned}
$$

on $\mathcal{M} \times \mathcal{M}$ relative to $\mathbf{P} \otimes P$. In particular, for every bounded measurable function $g$ on $\mathcal{M}$ we obtain

$$
\begin{aligned}
P(\{0\}) e^{\lambda(E)} \int \mathbf{P}(d \alpha) \varphi(\alpha, 0) g(\alpha) & =\int \bar{\Gamma}_{P}(d \alpha, d \mu) g(\alpha) 1\{\mu=0\} \\
& =\int P(d \mu) g(\mu)
\end{aligned}
$$

This shows that $P \ll \mathbf{P}$ with density $f(\mu)=P(\{0\}) e^{\lambda(E)} \varphi(\mu, 0)$.

From (10) we conclude further that the set $\left\{(x, \mu): f\left(\mu+\delta_{x}\right)>0=f(\mu)\right\}$ has measure zero relative to $\lambda \otimes \mathbf{P}=\Gamma_{\mathbf{P}}$. This means that for $\mathbf{P}$-almost all $\mu, f(\mu)=0$ whenever $f\left(\mu-\delta_{x}\right)=0$ for some $x \in \mu$. Hence $f$ has a version with increasing zero-set.

Remark 3.2 The preceding proof also shows that for each $\Lambda \in \mathcal{E}$ and $P$-almost all $\mu \in \mathcal{M}(E \backslash \Lambda)$, the conditional distribution of $P$ on $\mathcal{M}(\Lambda)$ given the configuration $\mu$ on $E \backslash \Lambda$ is absolutely continuous with respect to the restriction $\mathbf{P}_{1_{\Lambda} \lambda}$ of $\mathbf{P}$ to the state space $\Lambda$, and its density is given by the function $\alpha \rightarrow \varphi(\alpha, \mu) / \int \varphi(\cdot, \mu) d \mathbf{P}_{1_{\Lambda} \lambda}$. See [12] or Theorem 13.14 of [9]. 
Suppose now we are given an arbitrary measurable function $a: E \times \mathcal{M} \rightarrow[0, \infty[$. Can we define two prf's $P_{a}, P^{a} \in \mathcal{P}_{\lambda}$ such that the corresponding Papangelou intensities satisfy the inequality $\gamma_{P_{a}} \leq a \leq \gamma_{P^{a}}$ ? Eq. (10) suggests that we should define functions $\varphi_{a}, \varphi^{a}: \mathcal{M} \rightarrow\left[0, \infty\left[\right.\right.$ recursively by $\varphi_{a}(0)=\varphi^{a}(0)=1$ and

$$
\begin{aligned}
\varphi_{a}(\mu) & =\min _{x \in \mu} a\left(x, \mu-\delta_{x}\right) \varphi_{a}\left(\mu-\delta_{x}\right), \\
\varphi^{a}(\mu) & =\max _{x \in \mu} a\left(x, \mu-\delta_{x}\right) \varphi^{a}\left(\mu-\delta_{x}\right)
\end{aligned}
$$

when $\mu \neq 0$. We then have the following

Lemma 3.3 (a) $\varphi_{a}$ and $\varphi^{a}$ are measurable.

(b) If $a=\gamma_{Q}$ for some $Q \in \mathcal{P}_{\lambda}$ then $\varphi_{a}=\varphi^{a}=f_{Q} / f_{Q}(0)$.

(c) For all $\mu \in \mathcal{M}$ and $x \in E, \varphi_{a}\left(\mu+\delta_{x}\right) \leq a(x, \mu) \varphi_{a}(\mu)$ and $\varphi^{a}\left(\mu+\delta_{x}\right) \geq$ $a(x, \mu) \varphi^{a}(\mu)$.

(d) Suppose $\pi$ is a measurable map from $E$ into a second space $E^{\prime}$, and let $\pi^{*}$ : $\mu \rightarrow \mu \circ \pi^{-1}$ and $\pi^{\#}=\pi \times \pi^{*}:(x, \mu) \rightarrow\left(\pi x, \pi^{*} \mu\right)$ be the induced maps from $\mathcal{M}$ to $\mathcal{M}^{\prime}=\mathcal{M}\left(E^{\prime}\right)$ resp. from $E \times \mathcal{M}$ to $E^{\prime} \times \mathcal{M}^{\prime}$. If $a^{\prime}: E^{\prime} \times \mathcal{M}^{\prime} \rightarrow[0, \infty[$ is measurable and $a=a^{\prime} \circ \pi^{\#}$ then $\varphi_{a}=\varphi_{a^{\prime}} \circ \pi^{*}$ and $\varphi^{a}=\varphi^{a^{\prime}} \circ \pi^{*}$.

Proof. (a) The first equation of (11) can be written in the form $\varphi_{a}(\mu)=$ $\inf _{A \in \mathcal{E}_{0}} \varphi_{a, A}(\mu)$ for $\mu \neq 0$, where $\mathcal{E}_{0}$ is a countable basis of $E$ and

$$
\varphi_{a, A}(\mu)=\mu(A)^{-1} \int_{A} \mu(d x) a\left(x, \mu-\delta_{x}\right) \varphi_{a}\left(\mu-\delta_{x}\right)
$$

if $\mu(A)>0$ and $\varphi_{a, A}(\mu)=\infty$ otherwise. Since the mappings $(x, \mu) \rightarrow \mu-\delta_{x}$ on $\{(x, \mu): x \in \mu\}$ and $\mu \rightarrow \int \mu(d x) g(x, \mu)$ (for any measurable $g$ ) are measurable, we see by induction on $n$ that $\varphi_{a, A}$, and therefore $\varphi_{a}$, is measurable on the set $\left\{N_{E}=n\right\}$ of $n$-particle configurations. The measurability of $\varphi^{a}$ follows similarly.

(b) is obtained by comparing (11) with (9), (c) is evident, and (d) follows readily from (11) by induction on the particle number.

Suppose we know that $a \leq \gamma_{Q}$ for some $Q \in \mathcal{P}_{\lambda}$. Since then

$$
\varphi_{a} \leq \varphi^{a} \leq \varphi^{\gamma_{Q}}=f_{Q} / f_{Q}(0)
$$

by Lemma 3.3(b), it follows that $\varphi_{a}$ and $\varphi^{a}$ are $\mathbf{P}$-integrable. Their integrals are not less than $\mathbf{P}(\{0\})=e^{-\lambda(E)}$. Hence we can introduce the probability densities

$$
f_{a}=\varphi_{a} / \int \varphi_{a} d \mathbf{P}, \quad f^{a}=\varphi^{a} / \int \varphi^{a} d \mathbf{P}
$$

and the corresponding prf's

$$
P_{a}=f_{a} \mathbf{P}, \quad P^{a}=f^{a} \mathbf{P}
$$

which will be called the lower resp. upper prf for the function $a$. We will need the following continuity property of the mappings $a \rightarrow P_{a}$ and $a \rightarrow P^{a}$. 
Lemma 3.4 Suppose $a$ and $a_{k}, k \geq 1$, are nonegative functions on $E \times \mathcal{M}$ such that $a_{k} \leq \gamma_{Q}$ for some $Q \in \mathcal{P}_{\lambda}$ for all $k \geq 1$, and $a_{k} \rightarrow a \quad \lambda \otimes \mathbf{P}$-almost surely. Then $P_{a_{k}} \rightarrow P_{a}$ and $P^{a_{k}} \rightarrow P^{a}$ in total variation norm.

Proof. We only need to show that $\varphi_{a_{k}} \rightarrow \varphi_{a}$ and $\varphi^{a_{k}} \rightarrow \varphi^{a}$ in $L^{1}(\mathbf{P})-$ norm. This is because

$$
\left\|P_{a_{k}}-P_{a}\right\|=\int\left|f_{a_{k}}-f_{a}\right| d \mathbf{P} \leq 2 \int\left|\varphi_{a_{k}}-\varphi_{a}\right| d \mathbf{P} / \int \varphi_{a} d \mathbf{P}
$$

and $\int \varphi_{a} d \mathbf{P} \geq \mathbf{P}(\{0\})>0$, and similarly for the upper measures. In view of (12) and the dominated convergence theorem, it is sufficient to prove the almost-sure convergence. Let $C=\left\{\varphi_{a_{k}} \rightarrow \varphi_{a}\right.$ and $\left.\varphi^{a_{k}} \rightarrow \varphi^{a}\right\}$. We prove by induction on $n$ that $D_{n}=\left\{N_{E}=n\right\} \backslash C$ has $\mathbf{P}$-probability zero. The case $n=0$ is trivial. So let $n \geq 1$ and suppose that $\mathbf{P}\left(D_{n-1}\right)=0$. Let $\Delta=\left\{a_{k} \nrightarrow a\right\}$. By hypothesis, $\lambda \otimes \mathbf{P}(\Delta)=0$. Using (11) in the first step, we obtain

$$
\begin{aligned}
\mathbf{P}\left(D_{n}\right) & \leq \mathbf{P}\left(\mu \in \mathcal{M}: \exists x \in \mu \text { such that } \mu-\delta_{x} \in D_{n-1} \text { or }\left(x, \mu-\delta_{x}\right) \in \Delta\right) \\
& \leq \int \mathbf{P}(d \mu) \int \mu(d x)\left[1_{D_{n-1}}(\mu)+1_{\Delta}\left(x, \mu-\delta_{x}\right)\right] \\
& =\int \lambda(d x) \int \mathbf{P}(d \mu)\left[1_{D_{n-1}}(\mu)+1_{\Delta}(x, \mu)\right] \\
& =0
\end{aligned}
$$

This completes the induction step and the proof of the lemma.

\section{Proof of the theorem}

Let $P, Q \in \mathcal{P}_{\lambda}$ be two fixed prf's with Papangelou intensities $p=\gamma_{P}$ and $q=\gamma_{Q}$. We assume that (2) holds. To prove Theorem 1.1, we will first modify $p$ and $q$ by means of truncation and coarse-graining. The modified functions fail to satisfy (2), but a further modification will ensure that their lower resp. upper prf's satisfy (2). By the coarse-graining, we are then in the situation of a finite state space, so that the classical Holley-Preston inequality applies; cf. Remark 1.4. Finally we will use Lemma 3.4 to show that the lower and upper prf's converge to $P$ resp. $Q$ in suitable limits.

Entering into the details, we first assume without loss that $\lambda$ is a probability measure. (This is possible because multiplying $\lambda$ by a factor only changes the Papangelou intensities by the same factor.) Next, we fix two numbers $0<\delta<k<\infty$ and consider the truncated functions

$$
p_{k, \delta}=(p \wedge k) \vee \delta, \quad q_{k, \delta}=(q \wedge k) \vee \delta
$$

on $E \times \mathcal{M}$. The coarse-graining will be achieved by a finite measurable partition $\pi$ of $E$. We think of $\pi$ as a measurable mapping from $E$ onto $E^{\prime}=\{1, \ldots, m\}$ for a suitable number $m$. As in Lemma 3.3(d), we introduce the associated mapping $\pi^{*}: \mu \rightarrow \mu \circ \pi^{-1}$ 
from $\mathcal{M}$ into the set $\mathcal{M}^{\prime}=\mathcal{M}\left(E^{\prime}\right)$ of all finite point measures on $E^{\prime}$. Note that $\mathcal{M}^{\prime}$ is countable and its $\sigma$-algebra $\mathcal{F}^{\prime}$ consists of all subsets of $\mathcal{M}^{\prime}$.

Let $\mathcal{E}_{\pi}=\sigma(\pi)$ and $\mathcal{F}_{\pi}=\sigma\left(\pi^{*}\right)$ be the sub- $\sigma$-algebras of $\mathcal{E}$ resp. $\mathcal{F}$ which are generated by $\pi$ resp. $\pi^{*}$. We then form the conditional expectations

$$
p_{k, \delta, \pi}=\mathbb{E}_{\boldsymbol{\Gamma}}\left(p_{k, \delta} \mid \mathcal{E}_{\pi} \otimes \mathcal{F}_{\pi}\right) \quad q_{k, \delta, \pi}=\mathbb{E}_{\boldsymbol{\Gamma}}\left(q_{k, \delta} \mid \mathcal{E}_{\pi} \otimes \mathcal{F}_{\pi}\right)
$$

relative to the measure $\boldsymbol{\Gamma}=\lambda \otimes \mathbf{P}$, and we let $p_{k, \delta, \pi}^{\prime}$ and $q_{k, \delta, \pi}^{\prime}$ be the associated factorizations on $E^{\prime} \times \mathcal{M}^{\prime}$ satisfying $p_{k, \delta, \pi}=p_{k, \delta, \pi}^{\prime} \circ \pi^{\#}$ and $q_{k, \delta, \pi}=q_{k, \delta, \pi}^{\prime} \circ \pi^{\#}$. Here we use again the notation $\pi^{\#}=\pi \times \pi^{*}$. To ensure a version of (2) we finally replace $q_{k, \delta, \pi}$ by $\bar{q}_{k, \delta, \pi}=\bar{q}_{k, \delta, \pi}^{\prime} \circ \pi^{\#}$ with

$$
\bar{q}_{k, \delta, \pi}^{\prime}\left(x^{\prime}, \nu^{\prime}\right)=q_{k, \delta, \pi}^{\prime}\left(x^{\prime}, \nu^{\prime}\right) \vee \max _{\mu^{\prime} \leq \nu^{\prime}: x^{\prime} \notin \nu^{\prime}-\mu^{\prime}} p_{k, \delta, \pi}^{\prime}\left(x^{\prime}, \mu^{\prime}\right)
$$

$\left(x^{\prime}, \nu^{\prime}\right) \in E^{\prime} \times \mathcal{M}^{\prime}$. We consider the lower prf $P_{k, \delta, \pi}=P_{p_{k, \delta, \pi}}$ of $p_{k, \delta, \pi}$ and the upper $\operatorname{prf} \bar{Q}^{k, \delta, \pi}=P^{\bar{q}_{k, \delta, \pi}}$ for $\bar{q}_{k, \delta, \pi}$. These are well-defined because $p_{k, \delta, \pi} \leq \bar{q}_{k, \delta, \pi} \leq k=\gamma_{\mathbf{P}_{k \lambda}}$. Theorem 1.1 follows directly from the next two lemmas.

Lemma $4.1 \quad$ For all $k, \delta, \pi, P_{k, \delta, \pi} \preceq \bar{Q}^{k, \delta, \pi}$.

LEMma 4.2 In the limit when $\pi$ runs through a suitable sequence of partitions, $\delta \rightarrow 0$ and $k \rightarrow \infty, P_{k, \delta, \pi} \rightarrow P$ and $\bar{Q}^{k, \delta, \pi} \rightarrow Q$ in total variation norm.

Proof of Lemma 4.1. Let $h: \mathcal{M} \rightarrow \mathrm{R}$ be an increasing bounded measurable function, $h_{\pi}=\mathbb{E}_{\mathbf{P}}\left(h \mid \mathcal{F}_{\pi}\right)$ its conditional expectation, and $h_{\pi}^{\prime}: \mathcal{M}^{\prime} \rightarrow \mathrm{R}$ such that $h_{\pi}=h_{\pi}^{\prime} \circ \pi^{*}$.

1) $h_{\pi}^{\prime}$ is increasing. By the definition of $\mathbf{P}$, we have for each $\mu^{\prime} \in \mathcal{M}^{\prime}$

$$
h_{\pi}^{\prime}\left(\mu^{\prime}\right)=\mathbb{E} h\left(\sum_{i=1}^{m} \sum_{j=1}^{\mu^{\prime}(i)} \delta_{Z_{i, j}}\right),
$$

where $\left(Z_{i, j}\right)_{1 \leq i \leq m, j \geq 1}$ are independent random variables such that $Z_{i, j}$ has distribution $\lambda\left(\cdot \mid \pi^{-1}\{i\}\right)$. (We can assume without loss of generality that $\lambda\left(\pi^{-1}\{i\}\right)>0$ for all $i$.) The claim is thus obvious.

2) Let $P^{\prime}$ (resp. $\bar{Q}^{\prime}$ ) be the lower (resp. upper) prf on $\mathcal{M}^{\prime}$ for the function $p^{\prime}=$ $p_{k, \delta, \pi}^{\prime}$ (resp. $\bar{q}^{\prime}=\bar{q}_{k, \delta, \pi}^{\prime}$ ) relative to the Poisson prf $\mathbf{P}^{\prime}=\mathbf{P}_{\lambda^{\prime}}$ with intensity measure $\lambda^{\prime}=\lambda \circ \pi^{-1}$. Then $P^{\prime} \preceq \bar{Q}^{\prime}$. To see this we note first that $P^{\prime}, \bar{Q}^{\prime} \in \mathcal{P}_{\lambda^{\prime}}$ because $p^{\prime}$ and $\bar{q}^{\prime}$ are strictly positive. From Lemma 3.3 (c) and (15) we conclude that, for all $\mu^{\prime}, \nu^{\prime} \in \mathcal{M}^{\prime}$ with $\mu^{\prime} \leq \nu^{\prime}$ and all $x \notin \nu^{\prime}-\mu^{\prime}$,

$$
\gamma_{P^{\prime}}\left(x^{\prime}, \mu^{\prime}\right) \leq p^{\prime}\left(x^{\prime}, \mu^{\prime}\right) \leq \bar{q}^{\prime}\left(x^{\prime}, \nu^{\prime}\right) \leq \gamma_{\bar{Q}^{\prime}}\left(x^{\prime}, \nu^{\prime}\right) .
$$

That is, $P^{\prime}$ and $\bar{Q}^{\prime}$ satisfy condition (2). The assertion therefore follows from Remark 1.4 . 
3) To complete the proof we write

$$
\begin{aligned}
\int h d P_{k, \delta, \pi} & =\int \varphi_{p^{\prime}} \circ \pi^{*} h d \mathbf{P} / \int \varphi_{p^{\prime}} \circ \pi^{*} d \mathbf{P} \\
& =\int h_{\pi}^{\prime} d \mathbf{P}^{\prime} \\
& \leq \int h_{\pi}^{\prime} d \bar{Q}^{\prime}=\int h d \bar{Q}^{k, \delta, \pi} .
\end{aligned}
$$

In the above, we first used Lemma $3.3(\mathrm{~d})$. Then we replaced $h$ by $h_{\pi}$ and noticed that $\mathbf{P}^{\prime}$ is the $\pi^{*}$-image measure of $\mathbf{P}$. The inequality comes from steps 1 ) and 2), and the final equality is similar to the first two.

Proof of Lemma 4.2. 1) Let $P_{k, \delta}$ be the lower prf for $p_{k, \delta}$ and $Q^{k, \delta}$ the upper prf for $q_{k, \delta}$, cf. (14). Also, let $P_{k}$ be the lower prf for $p \wedge k$ and $Q^{k}$ the upper prf for $q \wedge k$. For given $k$, we have $p_{k . \delta} \leq q_{k, \delta} \leq k=\gamma_{\mathbf{P}_{k \lambda}}$ for all $\delta>0$. Lemma 3.4 therefore implies that $P_{k, \delta} \rightarrow P_{k}$ and $Q^{k, \delta} \rightarrow Q^{k}$ in total variation as $\delta \rightarrow 0$. Since $p_{k} \leq q_{k} \leq q=\gamma_{Q}$, Lemma 3.4 also shows that $P_{k} \rightarrow P$ and $Q^{k} \rightarrow Q$ in total variation as $k \rightarrow \infty$. We thus only need to find a sequence of partitions $\pi$ such that, for each $k, \delta, P_{k, \delta, \pi} \rightarrow P_{k, \delta}$ and $\bar{Q}^{k, \delta, \pi} \rightarrow Q^{k, \delta}$ as $\pi$ runs through this sequence. In view of the bound $p_{k, \delta, \pi} \leq \bar{q}_{k, \delta, \pi} \leq k$, Lemma 3.4 again tells us that we only need to show that $p_{k, \delta, \pi} \rightarrow p_{k, \delta}$ and $\bar{q}_{k, \delta, \pi} \rightarrow q_{k, \delta}$ $\boldsymbol{\Gamma}$-almost surely for a suitable sequence of $\pi$ 's. We will do this in the next two steps.

$2)$ By hypothesis on $E$ there exists a countable algebra $\mathcal{E}_{0}$ generating $\mathcal{E}$, and a routine argument shows that $\mathcal{F}=\sigma\left(N_{A}: A \in \mathcal{E}_{0}\right)$. Hence we can find an increasing sequence of finite measurable partitions $\pi$ of $E$ such that $\mathcal{E}_{\pi} \uparrow \mathcal{E}$ and $\mathcal{F}_{\pi} \uparrow \mathcal{F}$ as $\pi$ runs through this sequence. We simply write $\pi \rightarrow \infty$ for the limit along this sequence. Since $\mathcal{E}_{\pi} \otimes \mathcal{F}_{\pi} \uparrow \mathcal{E} \otimes \mathcal{F}$ as $\pi \rightarrow \infty$, the martingale convergence theorem implies that $p_{k, \delta, \pi} \rightarrow p_{k, \delta}$ and $q_{k, \delta, \pi} \rightarrow q_{k, \delta}$ as $\pi \rightarrow \infty$ with $\boldsymbol{\Gamma}$-probability 1 .

3) It remains to show that also $\bar{q}_{k, \delta, \pi} \rightarrow q_{k, \delta}$ as $\pi \rightarrow \infty$ with $\boldsymbol{\Gamma}$-probability 1. In view of (15) and the preceding step this will follow once we have shown that, for $\boldsymbol{\Gamma}$-almost all $(x, \nu)$,

$$
\limsup _{\pi \rightarrow \infty} \max p_{k, \delta, \pi}^{\prime}\left(\pi x, \mu^{\prime}\right) \leq q_{k, \delta}(x, \nu),
$$

where the maximum extends over all $\mu^{\prime} \in \mathcal{M}^{\prime}$ which are such that $\mu^{\prime} \leq \pi^{*} \nu$ and $\pi x \notin \pi^{*} \nu-\mu^{\prime}$. Each such $\mu^{\prime}$ is the $\pi^{*}$-image of some $\mu \in \mathcal{M}$ with $\mu \leq \nu$ and $x \notin \nu-\mu$, and then $p_{k, \delta, \pi}^{\prime}\left(\pi x, \mu^{\prime}\right)=p_{k, \delta, \pi}(x, \mu)$. Also, hypothesis (2) ensures that, for each such $\mu, p_{k, \delta}(x, \mu) \leq q_{k, \delta}(x, \nu)$. It is therefore sufficient to show that

$$
\boldsymbol{\Gamma}\left((x, \nu) \in E \times \mathcal{M}: \forall \mu \leq \nu, p_{k, \delta, \pi}(x, \mu) \rightarrow p_{k, \delta}(x, \mu) \text { as } \pi \rightarrow \infty\right)=1 .
$$

Let $\Delta=\left\{p_{k, \delta, \pi} \nrightarrow p_{k, \delta}\right.$ as $\left.\pi \rightarrow \infty\right\}$. We show by induction on $n$ that

$$
\boldsymbol{\Gamma}((x, \nu) \in E \times \mathcal{M}: \exists \alpha \leq \nu \text { s.t. } \alpha(E)=n \text { and }(x, \nu-\alpha) \in \Delta)=0 .
$$

For each $n \geq 0$, the left-hand side of (16) is equal to $\boldsymbol{\Gamma}\left(g_{n}>0\right)$, where $g_{0}=1_{\Delta}$ and

$$
g_{n}(x, \nu)=\int \nu(d y) g_{n-1}\left(x, \nu-\delta_{y}\right),
$$


$x \in E, \nu \in \mathcal{M}$. From Step 2) we know that $\int g_{0} d \boldsymbol{\Gamma}=0$, and the identity $\boldsymbol{\Gamma}=\lambda \otimes \mathbf{P}=\Gamma_{\mathbf{P}}$ implies that

$$
\begin{aligned}
\int g_{n} d \boldsymbol{\Gamma} & =\int \lambda(d x) \int \boldsymbol{\Gamma}(d y, d \nu) g_{n-1}(x, \nu) \\
& =\lambda(E) \int g_{n-1} d \boldsymbol{\Gamma}
\end{aligned}
$$

for all $n \geq 1$. This proves (16) and completes the proof of the lemma.

\section{References}

[1] Baddeley, A.J. and van Lieshout, M.N.M.(1995) Area-interaction point processes. Ann. Inst. Statist. Math. 46, 601-619.

[2] Batty, C.J.K. and Bollmann, H.W. (1980) Generalized Holley-Preston inequalities on measure spaces and their products. Z. Wahrscheinlichkeitstheorie Verw. Geb. 53, 157-174.

[3] Chayes, J.T., Chayes, L. and Kotecký, R. (1995) The analysis of the WidomRowlinson model by stochastic geometric methods. Commun. Math. Phys. 172, 551-569.

[4] Fortuin, C.M., Kasteleyn, P.W. and Ginibre, J. (1971) Correlation inequalities on some partially ordered sets. Commun. Math. Phys. 22, 89-103.

[5] Georgit, H.O. and Häggström, O. (1996) Phase transitions in continuum Potts models. Commun. Math. Phys. 181, 507-528.

[6] HARRIS, T.E. (1960) A lower bound for the critical probability in a certain percolation process. Proc. Cambridge Phil. Soc. 56, 13-20

[7] Holley, R. (1974) Remarks on the FKG inequalities. Commun. Math. Phys. 36, 227-232.

[8] Janson, S. (1984) Bounds on the distributions of extremal values of a scanning process. Stoch. Proc. Appl. 18, 313-328.

[9] Kallenberg, O. (1983) Random measures, 3rd edition. Akademie Verlag, Berlin.

[10] Lebowitz, J.L. And Monroe, J.L. (1972) Inequalities for higher order Ising spins and continuum fluids. Commun. Math. Phys. 28, 301-311.

[11] Lindvalu, T. (1992) Lectures on the coupling method. John Wiley, New York etc.

[12] Matthes, K., Warmuth, W. And Mecke, J. (1979) Bemerkungen zu einer Arbeit von Nguyen Xuan Xanh und Hans Zessin. Math. Nachr. 88, 117-127.

[13] Penrose, M.D. (1991) On a continuum percolation model. Adv. Appl. Probab. 23, 536556.

[14] Preston, C.J. (1974) A generalization of the FKG inequalities. Commun. Math. Phys. 36, 233-242.

[15] Preston, C.J. (1976) Spatial birth-and-death processes. Proc. 40th Sess. ISI (Warsaw 1975), Vol. 2. Bull. Inst. Internat. Statist. 46, 371-391.

[16] Ruelle, D. (1969) Statistical Mechanics. Rigorous results. Benjamin, New York Amsterdam.

[17] Widom, B. And Rowlinson, J.S. (1970) New model for the study of liquid-vapor phase transitions. J. Chem. Phys. 52, 1670-1684. 